\title{
CRESCIMENTO MICELIAL DE FUNGOS MICORRÍZICOS ARBUSCULARES E FORMAÇÃO DE MICORRIZA EM SOLO CONTAMINADO POR CÁDMIO
}

\author{
Growth mycelium of arbuscular mycorrhizal fungi and formation of mycorrbiza in soil contaminated by cadmium \\ Rúbia Marcia Siqueira Martins; Rogério Melloni2*; Eliane Guimarães Pereira Melloni ${ }^{3}$
${ }^{1}$ Bióloga; Mestre; Instituto de Recursos Naturais; Universidade Federal de Itajubá; e-mail (rubisiqueira@yahoo.com.br)
2 Professor; Instituto de Recursos Naturais; Universidade Federal de Itajubá; e-mail (*rogerio.melloni@gmail.com)
${ }_{3}^{3}$ Professora; Instituto de Recursos Naturais; Universidade Federal de Itajubá; e-mail (eliane.melloni@gmail.com)

Artigo enviado em 08/03/2017, aceito em 09/08/2017 e publicado em 29/09/2017.

Resumo - O mecanismo que envolve a ação de fungos micorrízicos arbusculares (FMAs) na proteção das plantas em solos contaminados tem sido objeto de estudo visando a sua utilização em áreas contaminadas por metais. Nesse sentido, este trabalho teve como objetivo analisar o crescimento micelial de FMAs e seu potencial de formação de micorriza em solos contaminados por cádmio. Para isso, foram instalados dois experimentos em casa de vegetação, em esquema bifatorial, sendo os fatores: a) quatro doses crescentes de Cd (0, 5, 10 e $\left.20 \mathrm{mg} \mathrm{kg}^{-1}\right)$ e b) três espécies de FMAs (Rhizophagus clarus, Gigaspora rosea e Acaulospora scrobiculata), e um controle, sendo três repetições por tratamento. Cada unidade experimental foi composta por um sistema construído em PVC, dividido em dois compartimentos por uma malha $40 \mu \mathrm{m}$, e mistura de solo e areia como substrato, com uma planta de milho em cada compartimento. A diferença entre os experimentos foi o local de infestação dos FMAs, sendo o experimento 1, infestado em compartimento contaminado por $\mathrm{Cd}$ e o experimento 2, infestado em compartimento não contaminado por $\mathrm{Cd}$. Em ambos os experimentos, avaliaram-se: porcentagem e intensidade de colonização, comprimento do micélio extrarradicular total e número de esporos. Todos os FMAs apresentaram comportamento diferenciado na produção de propágulos e formação de micorriza, em função das doses de Cd, nas duas posições de infestação. Verificou-se que houve crescimento micelial de todas as espécies de FMAs em solos contaminados e não contaminados por Cd, mostrando a capacidade desses FMAs crescerem em solos contaminados até $20 \mathrm{mg} \mathrm{kg}^{-1} \mathrm{de}$ Cd.

Palavras-Chave - contaminação do solo, propágulo, micélio, colonização micorrízica.

\begin{abstract}
The mechanism that involves the action of arbuscular mycorrhizal fungi (AMF) on the protection of plants in contaminated soils has been object of a study once its use aims in areas contaminated by metals. Thus, in this context, the purpose of this study was to analyze the mycelium growth of AMF and its potential of formation of mycorrhiza in soils contaminated by cadmium. For this, two experiments were performed in greenhouse conduction, using a bi-factorial scheme: a) four increasing doses of $\mathrm{Cd}\left(0,5,10 \mathrm{e} 20 \mathrm{mg} \mathrm{kg} \mathrm{kg}^{-1}\right)$ and b) three species of AMF (Rhizophagus clarus, Gigaspora rosea and Acaulospora scrobiculata), and one control, with three replicates per treatment. Each experimental unit consisted of a system constructed in PVC, divided into two compartments by a mesh screen $40 \mu \mathrm{m}$, and mixing of soil and sand as a substrate, with one corn plant in each compartment. The difference between two experiments was the place of AMF infestation, with the experiment 1 infected in compartment contaminated by $\mathrm{Cd}$ and the experiment 2 infected in compartment non contaminated by $\mathrm{Cd}$. In both experiments, there were analyzed: mycorrhizal colonization and intensity percentage, total extra radicular mycelium length and number of spores. All AMF presented different behavior in the production of propagules and formation of mycorrhiza, as a function of the doses of $\mathrm{Cd}$, in the two positions studied. It was verified that there was mycelium growth of the all AMF species in contaminated and non contaminated soils by $\mathrm{Cd}$, showing the capacity of these AMFs to growth in contaminated soils up to $20 \mathrm{mg} \mathrm{kg}^{-1}$ of $\mathrm{Cd}$.
\end{abstract}

Keywords - Soil contamination, propagule, mycelium, mycorrhizal colonization. 


\section{INTRODUÇÃO}

Alguns metais pesados, como o $\mathrm{Cu}, \mathrm{Ni}, \mathrm{Cr}$, $\mathrm{Fe}, \mathrm{Mn}$ e $\mathrm{Zn}$, são considerados essenciais para a nutrição dos organismos vivos, porém quando acumulados no solo, em elevadas concentrações, tornam-se tóxicos para a biota edáfica, com efeitos cumulativos na cadeia trófica, ocasionando, inclusive, riscos à saúde humana (PAIVA et al., 2004; ALVARENGA, 2013).

Entre os metais não essenciais ao metabolismo biológico e causadores de poluição do solo, destaca-se o cádmio, o qual apresentou um aumento de ocorrência significativo no meio ambiente, advindo das atividades antrópicas (OSKARSSON et al., 2004). O cádmio é amplamente encontrado na natureza e liberado pela intemperização de rochas magmáticas e sedimentares, emissões de gases de atividades vulcânicas, queimadas de florestas e transporte de materiais particulados (ZHAO et al., 2007; BURAK et al., 2010). Porém, a sua emissão por fontes antropogênicas originária de poluentes industriais, queima de combustíveis fósseis, uso de fertilizantes, atividades de mineração e disposição inadequadas de resíduos configura-se superior à contaminação natural (OSKARSSON et al., 2004; LU et al., 2012).

Devido ao aumento significativo da contaminação do solo por metais pesados, nas últimas décadas, diversas tecnologias que utilizam espécies de plantas e microrganismos capazes de extrair ou metabolizar elementos nocivos ao meio vêm sendo estudadas para a remediação de áreas impactadas, e são genericamente denominadas de fitorremediação (ANDRADE et al., 2009; MUGOUEI et al., 2011). Nos processos de fitorremediação, os poluentes inorgânicos como os metais pesados não podem ser decompostos, porém são estabilizados no solo ou permanecem retidos nos tecidos de plantas hiperacumuladoras (COBBETT; GOLDSBROUGH, 2002; PEREIRA et al., 2012). Nesse sentido, são diversos os mecanismos que regem o potencial de tolerância das espécies utilizadas no processo de fitorremediação do solo. A associação entre plantas e fungos micorrízicos arbusculares (FMAs) pode ser considerada como um dos mecanismos que contribui para essa tolerância a fatores de estresse. Porém, de acordo com Jentschke, Winter e Godbold (1999), Klauberg-Filho et al. (2005) e Del Ducca et al. (2015), elevada concentração de metais pesados disponíveis no solo pode ser considerada tóxica para esses fungos, alterando sua capacidade de associação e proteção às plantas.

Atualmente, o mecanismo que envolve a ação de FMAs na proteção das plantas em solos contaminados é conhecido como micorremediação, e tem sido objeto de estudo visando a sua utilização em programas de revegetação de áreas contaminadas por metais (NOGUEIRA, 2007; NOGUEIRA; SOARES, 2010). Apesar dos mecanismos do FMAs na estabilização dos metais pesados, ainda não estarem elucidados, pesquisas sugerem que existe maior retenção dessas substâncias no micélio fúngico, por adsorção e fixação em grânulos de polifosfato, protegendo a planta contra o excesso de metais e aumentando a estabilidade dos agregados no solo (HALL, 2002; OLGUÍN et al., 2007; KERBAUY, 2012).

A associação entre FMAs e plantas possui grande importância na competição, sucessão das espécies vegetais e na produtividade agrícola, por meio dos seus efeitos benéficos na nutrição e na otimização dos mecanismos de tolerância dessas plantas a fatores de estresses abióticos (MOREIRA; SIQUEIRA, 2006; COLODETE et al., 2014; VALADARES; MESCOLOTTI; CARDOSO, 2016). No entanto, há necessidade de se conhecer o desenvolvimento e distribuição de propágulos de FMAs em solos contaminados por metais pesados, principalmente por cádmio, os quais têm relação com a formação de micorriza e tolerância de seus hospedeiros diante desse contaminante. Esse conhecimento seria de fundamental importância quando do emprego de técnicas de micorremediação em solos contaminados por $\mathrm{Cd}$, já que poderia haver conecção por micélio entre áreas adjacentes contaminadas e não contaminadas por $\mathrm{Cd}$, com potencial de formação de micorriza nos hospedeiros utilizados nesse processo. Nesse pressuposto, esse trabalho teve como objetivo estudar o crescimento micelial dos FMAs e seu potencial de formação de micorriza em solos contaminados por cádmio, utilizando milho como planta-hospedeira.

\section{MATERIAL E MÉTODOS}

Dois experimentos foram instalados e conduzidos em casa de vegetação, com ventilação forçada de ar, sob temperatura controlada de $28^{\circ} \mathrm{C}$, da Universidade Federal de Itajubá, campus Itajubá (MG), sob as coordenadas $22^{\circ} 25^{\prime} 32^{\prime \prime}$ de latitude Sul, $45^{\circ} 27^{\prime} 10^{\prime \prime}$ de longitude Oeste e altitude de $856 \mathrm{~m}$. 
No total, os experimentos contaram com 75 unidades experimentais, descritas a seguir, as quais foram constituídas por sistemas de tubos de PVC de $100 \mathrm{~mm}$ - 4" de diâmetro, compostos por dois compartimentos de $15 \mathrm{~cm}$ cada. Entre os compartimentos foi inserida uma tela de poliéster, com abertura de malha de $40 \mu \mathrm{m}$, conforme recomendação de Cruz e Martins (1998), para permitir a movimentação de hifas de FMAs e impedir a passagem de raízes.

Cada compartimento foi preenchido com substrato originado de amostra de Latossolo vermelho distrófico típico peneirado em malha de 2 $\mathrm{mm}$ e areia média, na proporção de 1:1, esterilizado em autoclave por $1 \mathrm{~h}$ a $121^{\circ} \mathrm{C}$. A análise física $\mathrm{e}$ química foi feita segundo recomendações da Embrapa (1997) e os resultados encontram-se na tabela 1.

Para o experimento 1, foi empregado delineamento inteiramente casualizado (DIC), em esquema bifatorial $4 \times 3$, sendo $\mathrm{O}$ fator 1 correspondente a quatro doses de cádmio: $(0,5,10$ e $20 \mathrm{mg} \mathrm{kg}^{-1}$ ), de acordo com Cunha et al. (2008), e o fator 2 a três espécies de FMAs: Rhizophagus clarus, Gigaspora rosea e Acaulospora scrobiculata, com três repetições por tratamento, totalizando 36 unidades experimentais. Para o experimento 2, também foi foi empregado delineamento inteiramente casualizado (DIC), em esquema bifatorial $3 \times 3$, sendo o fator 1 correspondente a três doses de cádmio: (5, 10 e 20 $\mathrm{mg} \mathrm{kg}{ }^{-1}$ ), de acordo com Cunha et al. (2008), e o fator 2 a três espécies de FMAs: Rhizophagus clarus, Gigaspora rosea e Acaulospora scrobiculata, com três repetições por tratamento, totalizando 27 unidades experimentais. Foi empregado, também, um tratamento controle, contendo as quatro doses de cádmio, sem FMAs e com três repetições, totalizando 12 unidades experimentais.

A diferença entre os dois experimentos foi quanto ao local de infestação do solo com propágulos de FMAs, ora em compartimento não contaminado (Experimento 1 - local 1) e ora em compartimento contaminado por Cd (Experimento 2 - local 2), conforme figura 1 , de modo a avaliar $O$ crescimento/deslocamento micelial. Essa infestação foi feita com porções de terra e propágulos de FMAs das espécies - Rhizophagus clarus, Gigaspora rosea, Acaulospora scrobiculata, obtidos da ESALQ/USP, de forma a aplicar individualmente 100 esporos por planta, conforme os tratamentos supracitados.

Tabela 1. Composição física e química de amostra do Latossolo vermelho distrófico típico utilizado na montagem dos experimentos.

\begin{tabular}{clccccc}
\hline $\begin{array}{c}\text { Areia grossa } \\
(\mathrm{kg} / \mathrm{kg})\end{array}$ & $\begin{array}{c}\text { Areia fina } \\
(\mathrm{kg} / \mathrm{kg})\end{array}$ & $\begin{array}{c}\text { Silte } \\
(\mathrm{kg} / \mathrm{kg})\end{array}$ & $\begin{array}{c}\text { Argila } \\
(\mathrm{kg} / \mathrm{kg})\end{array}$ & $\begin{array}{c}\text { Classificação } \\
\text { textural }\end{array}$ & $\begin{array}{c}\text { Tipo de } \\
\text { Solo }\end{array}$ & $\begin{array}{c}\mathrm{pH} \\
\mathrm{H}_{2} \mathrm{O}\end{array}$
\end{tabular}

\begin{tabular}{|c|c|c|c|c|c|c|}
\hline 0,166 & 0,115 & 0,117 & 0,602 & Muito argilosa & 3 & 4,65 \\
\hline $\begin{array}{c}\mathbf{P} \\
\left(\mathrm{mg} / \mathrm{dm}^{3}\right)\end{array}$ & $\begin{array}{c}\mathbf{K} \\
\left(\mathrm{mg} / \mathrm{dm}^{3}\right)\end{array}$ & $\begin{array}{c}\mathbf{N a} \\
\left(\mathrm{mg} / \mathrm{dm}^{3}\right)\end{array}$ & $\begin{array}{c}\mathbf{C a}^{2+} \\
\left(\mathrm{cmol}_{\mathrm{c}} / \mathrm{dm}^{3}\right)\end{array}$ & $\begin{array}{c}\mathbf{M g}^{2+} \\
\left(\mathrm{cmol}_{\mathrm{c}} / \mathrm{dm}^{3}\right)\end{array}$ & $\begin{array}{c}\mathbf{A l}^{3+} \\
\left(\mathrm{cmol}_{\mathrm{c}} / \mathrm{dm}^{3}\right)\end{array}$ & $\begin{array}{c}\mathbf{H}+\mathbf{A l} \\
\left(\mathrm{cmol}_{\mathrm{c}} / \mathrm{dm}^{3}\right)\end{array}$ \\
\hline 0,6 & 5 & 7,5 & 0,26 & 0,04 & 1,7 & 7,4 \\
\hline $\begin{array}{c}\mathrm{SB} \\
\left(\mathrm{cmol}_{\mathrm{c}} / \mathrm{dm}^{3}\right)\end{array}$ & $\begin{array}{c}\mathbf{t} \\
\left(\mathrm{cmol}_{\mathrm{c}} / \mathrm{dm}^{3}\right)\end{array}$ & $\begin{array}{c}\mathbf{T} \\
\left(\mathrm{cmol}_{\mathrm{c}} / \mathrm{dm}\right)\end{array}$ & $\begin{array}{c}\mathbf{V} \\
(\%)\end{array}$ & $\begin{array}{c}\mathbf{M} \\
(\%)\end{array}$ & $\begin{array}{c}\text { ISNa } \\
(\%)\end{array}$ & $\begin{array}{c}\text { MO } \\
\text { (dag/kg) }\end{array}$ \\
\hline 0,35 & 2,05 & 7,75 & 4,5 & 82,9 & 0,42 & 2,5 \\
\hline $\begin{array}{l}\text { P-Rem } \\
\text { (mg/L) }\end{array}$ & $\begin{array}{c}\mathbf{C u} \\
\left(\mathrm{mg} / \mathrm{dm}^{3}\right)\end{array}$ & $\begin{array}{c}\mathbf{M n} \\
\left(\mathrm{mg} / \mathrm{dm}^{3}\right)\end{array}$ & $\begin{array}{c}\mathbf{F e} \\
\left(\mathrm{mg} / \mathrm{dm}^{3}\right)\end{array}$ & $\begin{array}{c}\mathbf{Z n} \\
\left(\mathrm{mg} / \mathrm{dm}^{3}\right)\end{array}$ & - & - \\
\hline 10,3 & 1,5 & 0,6 & 21,8 & 1,93 & - & - \\
\hline
\end{tabular}




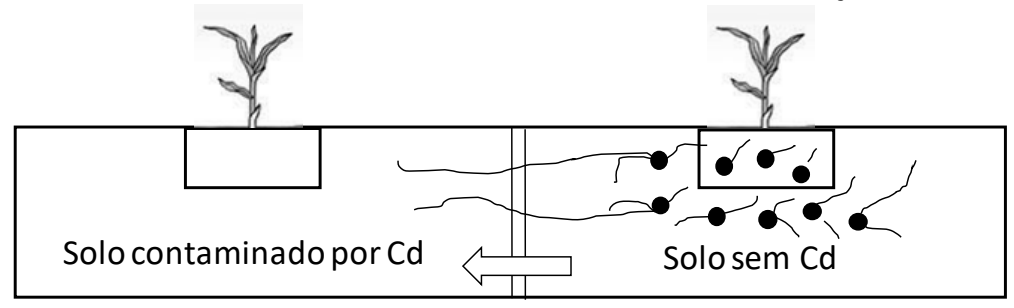

Experimento 2

Local de infestação 2

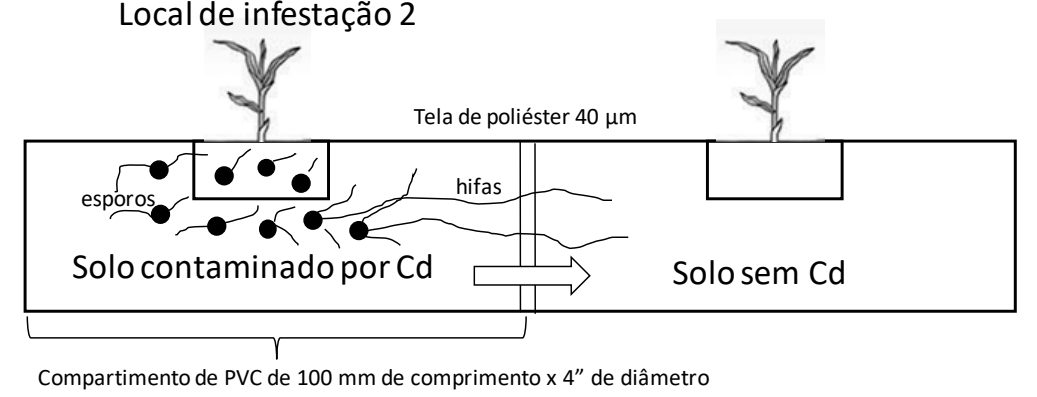

Figura 1. Ilustração diferenciando o local de infestação do substrato com fungos micorrízicos arbusculares nos dois experimentos, em local não contaminado (Experimento 1) ou contaminado por cádmio (Experimento 2).

Inicialmente, em cada compartimento foram semeadas três sementes de milho (Zea mays L.), cultivar Monsanto DKB, previamente desinfestadas com álcool a $70 \%$ e hipoclorito de sódio a $1 \%$, de acordo com Lazarotto, Muniz e Santos (2010). Após 11 dias de semeadura, as plantas foram desbastadas, deixando-se apenas uma por compartimento.

Aos 50 dias após a infestação do substrato, as plantas de cada compartimento, em ambos os experimentos, foram coletadas para a realização das análises descritas a seguir.

A colonização radicular foi determinada de duas formas, por meio da intensidade e da porcentagem, após coloração das raízes conforme Phillips e Haymann (1970). A intensidade de colonização radicular envolve metodologia proposta Bethlenfalvay et al. (1981), cuja leitura da ocupação da área radicular, variando de 0 a $100 \%$, é feita por meio de segmentos dispostos em lâmina de microscopia. Já a porcentagem de colonização radicular é determinada pela observação da ocorrência de formação de micorriza em segmentos de raízes dispostos em placa quadriculada, conforme Giovanetti e Mosse (1980). Ambas as metodologias descrevem a ocupação das raízes pelos FMAs, sendo a primeira representando a intensidade individual dessa ocupação e a segunda o número de raízes micorrizadas, independentemente do grau de ocupação.

A determinação do comprimento do micélio extrarradicular total de FMAs no substrato foi realizada de acordo com o método descrito por Melloni e Cardoso (1999). O número de esporos de FMAs nas amostras do substrato foi determinada seguindo-se a metodologia proposta por Gerdemann e Nicholson (1963), utilizando amostras de $50 \mathrm{~mL}$ de solo.

Os dados obtidos da intensidade de colonização, porcentagem de colonização, comprimento do micélio extrarradicular total e número de esporos foram submetidos ao teste de normalidade por Shapiro-Wilk a $95 \%$ de significância e à análise de variância (ANOVA), utilizando-se o software SISVAR, versão 5.3 (FERREIRA, 2011), 
para avaliação das fontes de variação dos dois fatores - cádmio e FMA, em ambos os experimentos. Quando da interação entre os fatores, os FMAs foram avaliados em cada dose de $\mathrm{Cd}$ por meio de comparação de médias pelo teste de Tukey a 5\%, enquanto o comportamento dos FMAs ao longo das doses de $\mathrm{Cd}$ foi avaliado por meio de análises de regressão, pelo mesmo software.

\section{RESULTADOS E DISCUSSÃO}

Experimento 1 - Crescimento micelial e formação de micorriza em solo contaminado por Cd

A porcentagem de colonização (Figura 2A) variou em função do FMA, com comportamento semelhante entre as espécies Acaulospora scrobiculata, Rhirophagus clarus e Gigaspora rosea, nas doses 0, 10, 20 $\mathrm{mg} \mathrm{kg-1}$, mas com maior valor para Acaulospora scrobiculata na dose $5 \mathrm{mg} \mathrm{kg}^{-1}$. Também houve efeito diferenciado dos FMAs quanto à intensidade de colonização (Figura 2B), sem padrão de resposta ao longo das doses, ora com maior valor para Acaulospora scrobiculata (nas doses 0 e $10 \mathrm{mg} \mathrm{kg}^{-1}$ ) e ora menor valor na dose $5 \mathrm{mg} \mathrm{kg}^{-1}$.

Apesar da reduzida taxa de colonização do hospedeiro no compartimento contaminado por $\mathrm{Cd}$ (em torno de $25 \%$ de raízes colonizadas com até $3 \%$ de intensidade), por meio desses resultados (Figuras $2 \mathrm{~A}$ e $2 \mathrm{~B}$ ) pode-se verificar que todas as espécies de FMAs apresentaram capacidade de formação de micorriza, mesmo em solos contaminados por até 20 $m g \mathrm{~kg}^{-1}$ de Cd. Salienta-se que o tempo de condução do experimento foi de 50 dias, suficiente para a micorrização, mas com baixos valores, conforme também observado previamente por Del Ducca et al. (2015), ao estudarem o efeito das diferentes doses de $\mathrm{Cd}$ na formação de micorriza em milhos transgênico e não transgênico.

Quanto ao comprimento de micélio extrarradicular (Figura 2C), observado no compartimento contaminado, também houve variação de comportamento dos FMAs, sendo Gigaspora rosea o que promoveu maior formação em relação às outras espécies nas doses 0 e $5 \mathrm{mg} \mathrm{kg}^{-1}$. $\mathrm{Na}$ dose $10 \mathrm{mg} \mathrm{kg}^{-1}$, Rhizophagus clarus promoveu maior comprimento de micélio e na dose mais elevada (20 $\mathrm{mg} \mathrm{kg}{ }^{-1}$ ), a espécie Acaulospora scrobiculata produziu o maior comprimento de micélio em relação às outras espécies.
Assim, o crescimento de micélio extrarradicular observado no compartimento de solo contaminado por $\mathrm{Cd}$, é muito importante em ambientes sob esse interferente, visto ser essa estrutura a responsável pela ocupação do solo e formação de estruturas reprodutivas, como esporos e colonização de hospedeiros. Segundo Moreira e Siqueira (2006), Cardoso et al. (2010) e Valadares, Mescolotti e Cardoso (2016), o micélio extrarradicular, além de garantir o aumento da absorção de nutrientes pelas raízes das plantas, é o responsável pelas alterações fisiológicas nas raízes, modificações na rizosfera e estabilidade dos agregados do solo, a qual está relacionada diretamente ao comprimento do micélio fúngico.

Quanto ao número de esporos formados no compartimento contaminado por Cd (Figura 2D), todas as espécies de FMAs também apresentaram comportamento diferenciado entre as doses, exceto para as maiores doses (10 e $\left.20 \mathrm{mg} \mathrm{kg}^{-1}\right)$, sem diferença entre os FMAs. No entanto, na dose $0 \mathrm{mg}$ $\mathrm{kg}^{-1}$, a Acaulospora scrobiculata promoveu menor produção de esporos em relação às outras espécies e na dose $5 \mathrm{mg} \mathrm{kg}^{-1}$, Rhizophagus clarus se destacou com a maior produção.

Apesar dos efeitos do excesso de metais sobre os FMAs ainda não estarem elucidados, Klauberg-Filho et al. (2005), Amir et al. (2013) e Yang et al. (2015) sugerem que altas concentrações de metais no solo podem reduzir o crescimento micelial, o grau de colonização e a esporulação dos FMAs, pois sua toxicidade pode causar a inibição da germinação e do desenvolvimento inicial das hifas fúngicas, atrasando ou suprimindo a formação da micorriza e comprometendo a germinação dos esporos.

No entanto, o resultado obtido (Figura 2) comprova a capacidade das espécies de FMAs utilizadas (Acaulospora scrobiculata, Rhizophagus clarus e Gigaspora rosea) em produzir micélio mesmo em solos contaminados por $\mathrm{Cd}$, sem comprometimento de sua capacidade de formação de micorriza em hospedeiros cultivados nesses ambientes. Essa habilidade pode ser considerada fundamental ao sucesso de programas de recuperação de áreas contaminadas por $\mathrm{Cd}$, utilizando micorremediação como estratégia. Esse resultado corrobora outros propostos por Nogueira e Soares (2010), Soares e Carneiro (2010) e Garcia et al. (2016), os quais indicaram a utilização de FMAs em solos degradados e contaminados por metais.

Klauberg-Filho, Siqueira e Moreira (2002) concluíram, em estudo com solos contaminados, que 
os FMAs e sua simbiose ocorrem de forma generalizada até mesmo em solo com elevada concentração de $\mathrm{Zn}$, Cd, Cu e Pd, porém os metais pesados podem exercer efeitos diferentes sobre os FMAs de acordo com o grau de poluição, reduzindo a diversidade e aumentando a dominância de espécies mais adaptadas. Sendo assim, Nogueira e Soares (2010) e Garcia et al. (2016) preconizaram que, para garantir o sucesso da reabilitação de solos contaminados por metais, utilizando FMAs, deve-se providenciar isolados fúngicos adaptados às

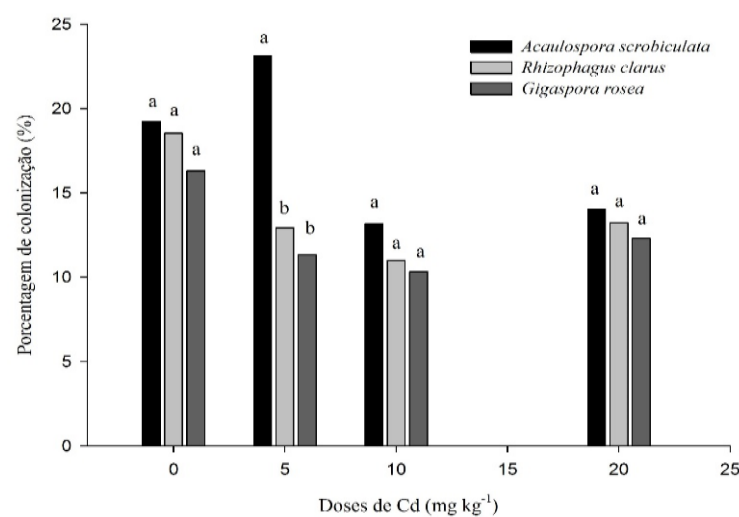

A

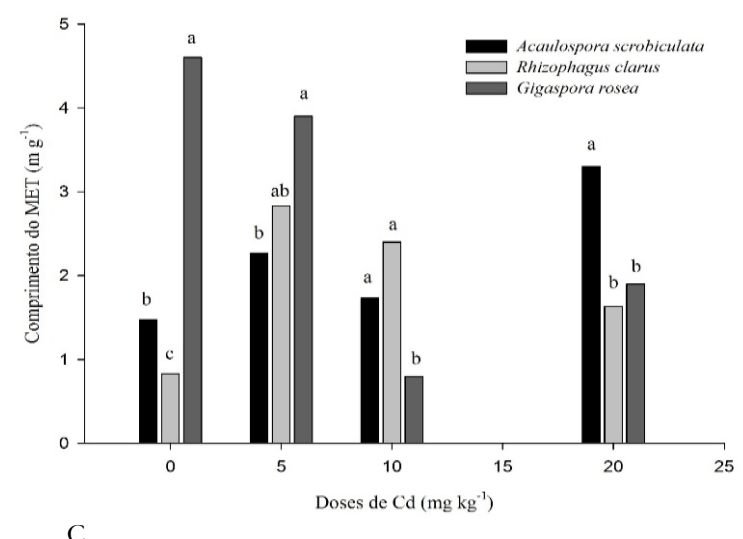

condições excessivas do contaminante, à planta hospedeira e às condições edafoclimáticas. Para isso, os mesmos citam a importância de estudos para a compreensão dos mecanismos de retenção e compartimentalização desses elementos contaminantes em raízes micorrizadas e hifas fúngicas.

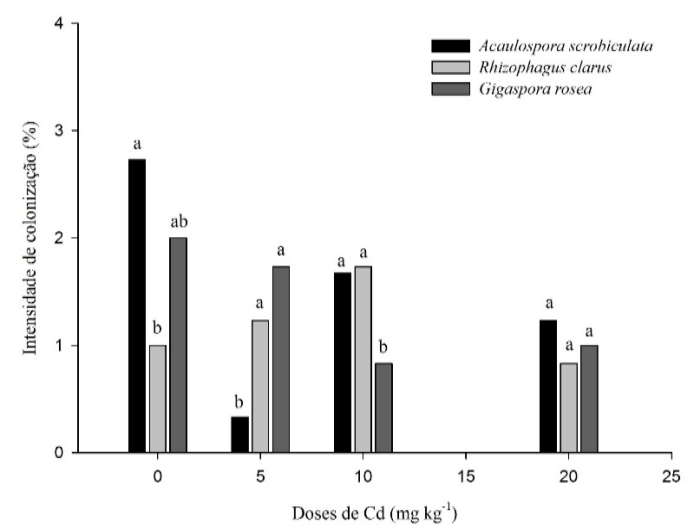

B

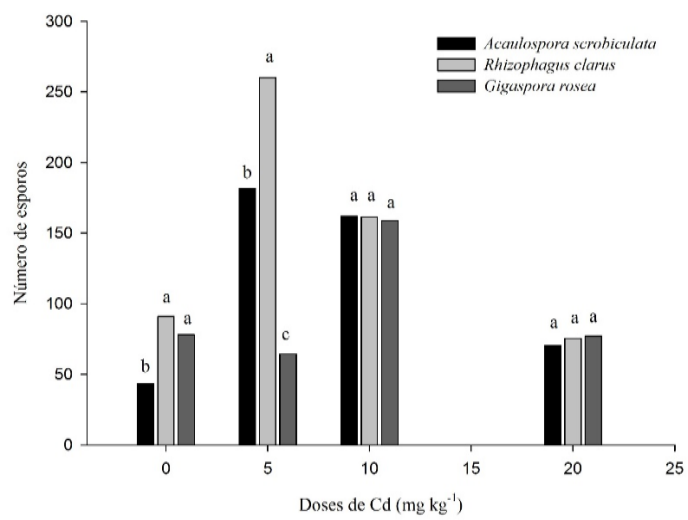

Figura 2. Colonização radicular, dada pela porcentagem (A) e intensidade (B), comprimento de micélio extrarradicular (C) e número de esporos (D) das diferentes espécies de fungos micorrízicos arbusculares, no compartimento com solo contaminado por $\mathrm{Cd}$. Médias seguidas por letras iguais na mesma dose não diferem estatisticamente entre si, por Tukey a 5\% de significância.

Lins et al. (2006), Silva et al. (2006), Colodete, Dobbs e Ramos (2014) enfatizaram que os FMAs inoculados em solos contaminados por metais podem atuar no reestabelecimento de plantas fitoestabilizadoras e, ainda, de acordo com Nogueira e Soares (2010), o grande potencial do FMA em programas de fitorremediação se dá pelo auxílio ao estabelecimento da planta hospedeira em ambiente degradado, por meio do seu papel nutricional e por sua atuação como agente tamponante, a qual reduziria a absorção do metal pela planta. 
Os dados do comportamento diferenciado das espécies Acaulospora scrobiculata, Rhizophagus clarus e Gigaspora rosea deslocadas para a região contaminada por Cd encontram-se na figura 3. Para porcentagem de colonização radicular (Figura 3A) apenas a espécie Acaulospora scrobiculata apresentou redução linear dos valores ao longo das doses de $\mathrm{Cd}$, demonstrando maior sensibilidade dessa espécie em elevadas doses do contaminante. Para as demais espécies, o efeito foi variável, sendo reduzida até a dose $10 \mathrm{mg} \mathrm{kg}^{-1}$, e incremento a partir dessa dose. Todas as espécies apresentaram comportamento quadrático para a intensidade de colonização (Figura 3B), porém, contrariamente às outras espécies, onde se observou redução ao longo das doses de $\mathrm{Cd}$, Rhizophagus clarus apresentou aumento da colonização até a dose $10 \mathrm{mg}$ $\mathrm{kg}^{-1} \mathrm{com}$ redução na dose $20 \mathrm{mg} \mathrm{kg}^{-1}$. Acaulospora scrobiculata apresentou comportamento descrito como linear e positivo para comprimento de micélio extrarradicular (Figura 3C), enquanto houve variação de comportamento para os demais FMAs, com quedas de diferentes intensidades ao longo das doses de Cd para Rhirophagus clarus e Gigaspora rosea. Quanto ao número de esporos (Figura 3D), todas as espécies exibiram comportamento quadrático, com aumento do valor até a dose $10 \mathrm{mg} \mathrm{kg}^{-1}$, e redução em seguida. Foi evidente a redução da esporulação na última dose (20 mg kg-1), independentemente da espécie fúngica. Tal resultado mostra a sensibilidade dessas espécies na produção de esporos, o que comprometeria a estratégia de sobrevivência quando em ambientes contaminados por $\mathrm{Cd}$.

No entanto, apesar do comprometimento na formação de estruturas importantes como essas, em ambientes contaminados por $\mathrm{Cd}$, mesmo em doses acima de $10 \mathrm{mg} \mathrm{kg}^{-1}$, as espécies fúngicas estudadas mantiveram a capacidade de produzi-las e de colonizarem os hospedeiros.

Klauberg-Filho, Siqueira e Moreira (2002) concluíram, em estudo com solos contaminados, que os FMAs e sua simbiose ocorrem de forma generalizada até mesmo em solo com elevada concentração de $\mathrm{Zn}, \mathrm{Cd}$, Cu e Pd, porém os metais pesados podem exercer efeitos diferentes sobre os FMAs de acordo com o grau de contaminação, reduzindo a diversidade e aumentando a dominância de espécies mais adaptadas.
Experimento 2 - Crescimento micelial e ormação de micorriza em solo não contaminado por $\mathrm{Cd}$

Houve crescimento micelial para o compartimento de solo não contaminado por $\mathrm{Cd}$ e formação de micorriza em milho. Os resultados da porcentagem e intensidade de colonização radicular, comprimento do micélio extrarradicular total e número de esporos entre as espécies de FMAs deslocadas das regiões contaminadas por $\mathrm{Cd}$ para as regiões não contaminadas encontram-se na figura 4 .

A porcentagem de colonização radicular variou em função do FMA (Figura 4A), sendo o maior valor para Acaulospora scrobiculata nas doses 5 e $20 \mathrm{mg} \mathrm{kg}^{-1}$ e para Rhizophagus clarus e Gigaspora rosea na dose $10 \mathrm{mg} \mathrm{kg}^{-1}$.

Também houve diferença de resposta dos FMAs quanto à intensidade de colonização (Figura 4B), com maior valor para Acaulospora scrobiculata na dose $0 \mathrm{mg} \mathrm{kg}^{-1}$, maior para Gigaspora rosea na dose 5 $\mathrm{mg} \mathrm{kg}{ }^{-1}$, e comportamento inverso entre as espécies Acaulospora scrobiculata e Gigaspora rosea, nas doses 10 e $20 \mathrm{mg} \mathrm{kg}^{-1}$. Dessa forma, novamente, apesar das taxas de colonização serem baixas, em torno de $20 \%$ de raízes colonizadas (Figura 4A) com até $3 \%$ de intensidade (Figura 3B), pode-se verificar que os FMAs produziram micélio da região contaminada por Cd para a região não contaminada, onde promoveram a formação de micorriza no milho. Esse resultado comprova, novamente, a habilidade dessas espécies em se propagar por solos contaminados por $\mathrm{Cd}$ e colonizar hospedeiros que se encontram em solos não contaminados limítrofes.

Essa capacidade fúngica de formar micorriza se deve à extensão de hifas desenvolvidas, com comportamento diferenciado entre as espécies de FMAs. Assim, para comprimento de micélio extrarradicular total (Figura 4C), a espécie Gigaspora rosea apresentou maior valor na dose $0 \mathrm{mg} \mathrm{kg}^{-1}$. $\mathrm{Na}$ dose $5 \mathrm{mg} \mathrm{kg}^{-1}$ a Acaulospora scrobiculata promoveu menor formação e nas doses $10 \mathrm{mg} \mathrm{kg}^{-1}$ e $20 \mathrm{mg} \mathrm{kg}^{-1}$ a Acaulospora scrobiculata e Gigaspora rosea demostraram maiores valores em relação àquele promovido por Rhizophagus clarus. Novamente, os valores estiveram na ordem de $3 \mathrm{~m} \mathrm{~g}^{-1}$ de solo, próximos aos observados no compartimento contaminado por $\mathrm{Cd}$ (Figura 2C) e aos encontrados por Del Ducca et al. (2015), conforme já discutido.

Para o número de esporos (Figura 4D), também houve diferença entre as espécies de FMAs nas diferentes doses, sendo que na dose $0 \mathrm{mg} \mathrm{kg}^{-1}$, Acaulospora scrobiculata apresentou os menores valores. 
$\mathrm{Na}$ dose $5 \mathrm{mg} \mathrm{kg}^{-1}$ Gigaspora rosea apresentou a maior formação de esporos, e na dose $20 \mathrm{mg} \mathrm{kg}^{-1}$ a espécie Rhizophagus clarus apresentou maior número de esporos em relação às demais. Klauberg-Filho, Siqueira e Moreira (2002) já haviam demonstrado, em estudo sobre a ocorrência e a diversidade de FMA sem solos contaminados por $\mathrm{Zn}, \mathrm{Cd}$, $\mathrm{Cu}$ e $\mathrm{Pb}$, que as espécies Acaulospora mellea e Rhizophagus clarus dominaram a população de esporos encontrada, indicando o maior potencial de tolerância dessas espécies ao excesso de metais pesados. A maior esporulação e potencial de adaptação do Rhizophagus clarus em área contaminada também pode ser comprovado, segundo Cabral et al. (2010), diante da maior capacidade de retenção de $\mathrm{Cu}, \mathrm{Cd}$ e $\mathrm{Pb}$ em seu micélio fúngico.

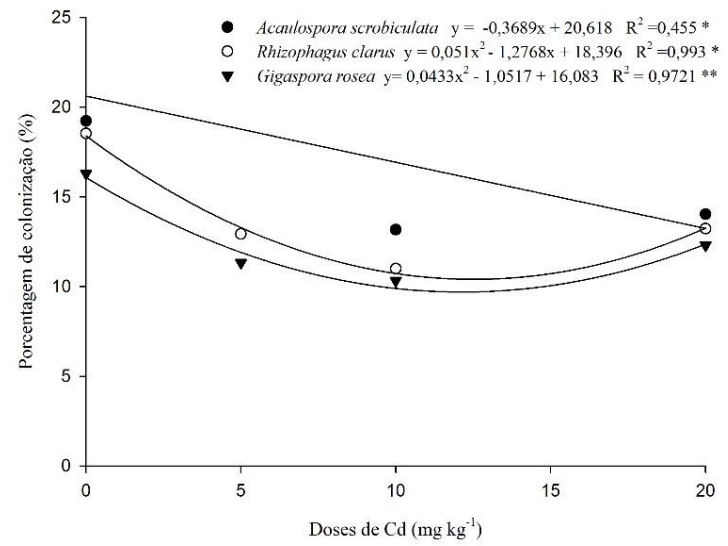

A

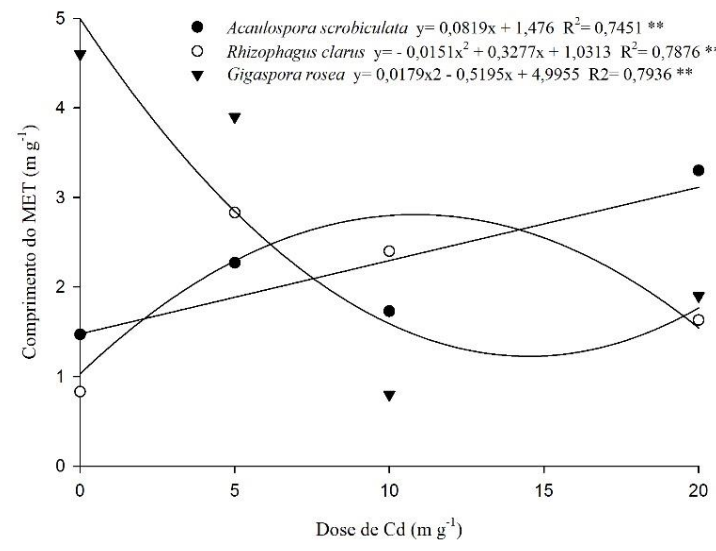

$\mathrm{C}$

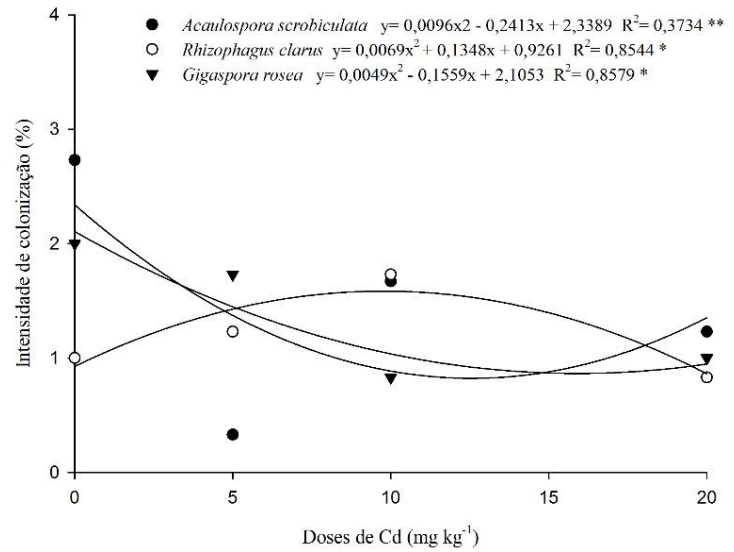

B

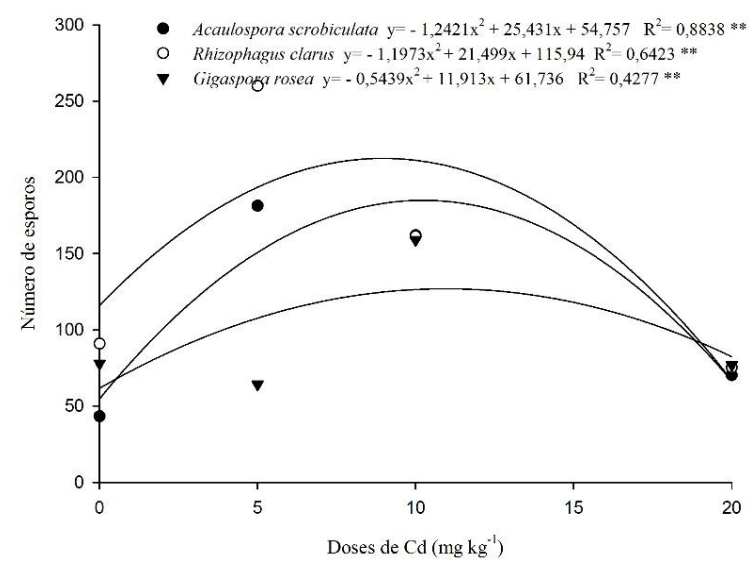

D

Figura 3. Comportamento das espécies Acaulospora scrobiculata, Rhizophagus clarus e Gigaspora rosea deslocadas para a região contaminada por $\mathbf{C d}$, em relação às doses de Cd. A - Porcentagem de colonização. B - Intensidade de colonização. C - Comprimento do micélio extrarradicular. D - Número de esporos. * $=\mathrm{p}<0,05 ; * *=\mathrm{p}<0,01$ 


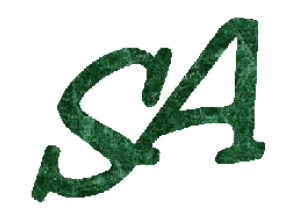

REVISTA SCIENTIA AGRARIA

Versão On-line ISSN 1983-2443

Versão Impressa ISSN 1519-1125

SA vol. $18 \mathrm{n}^{\circ} .3$ Curitiba Jul/Set. 2017 p. 48-60
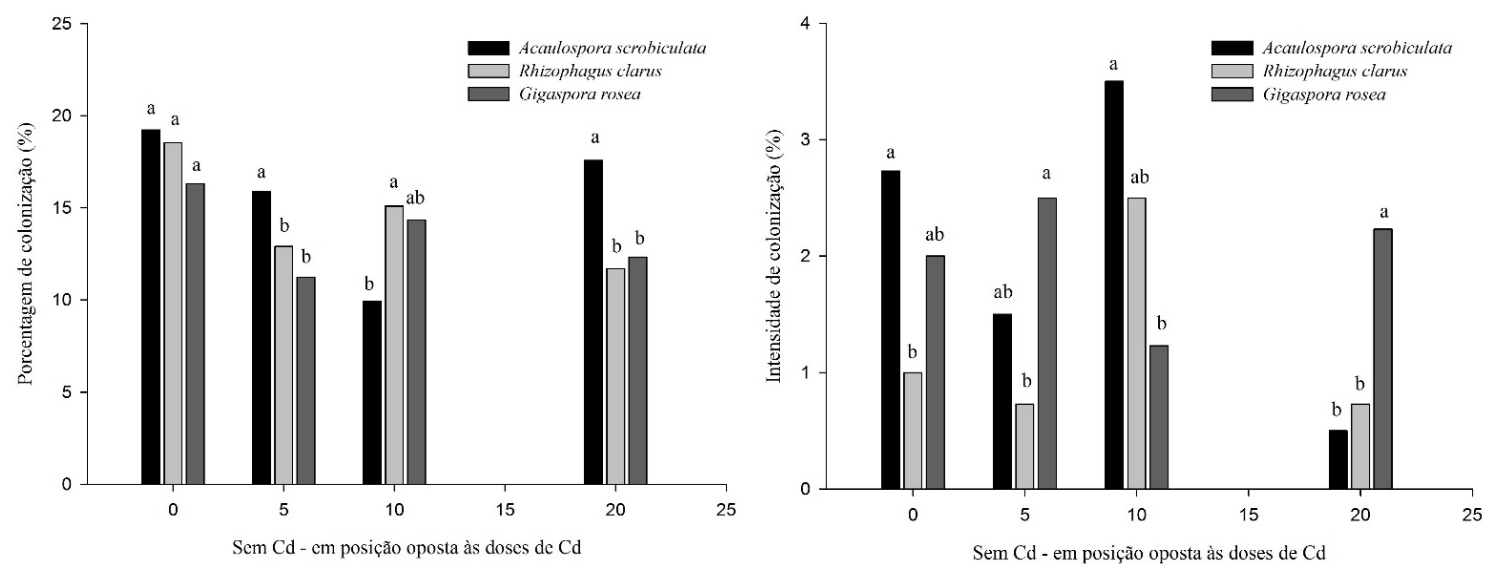

A

$\mathrm{B}$
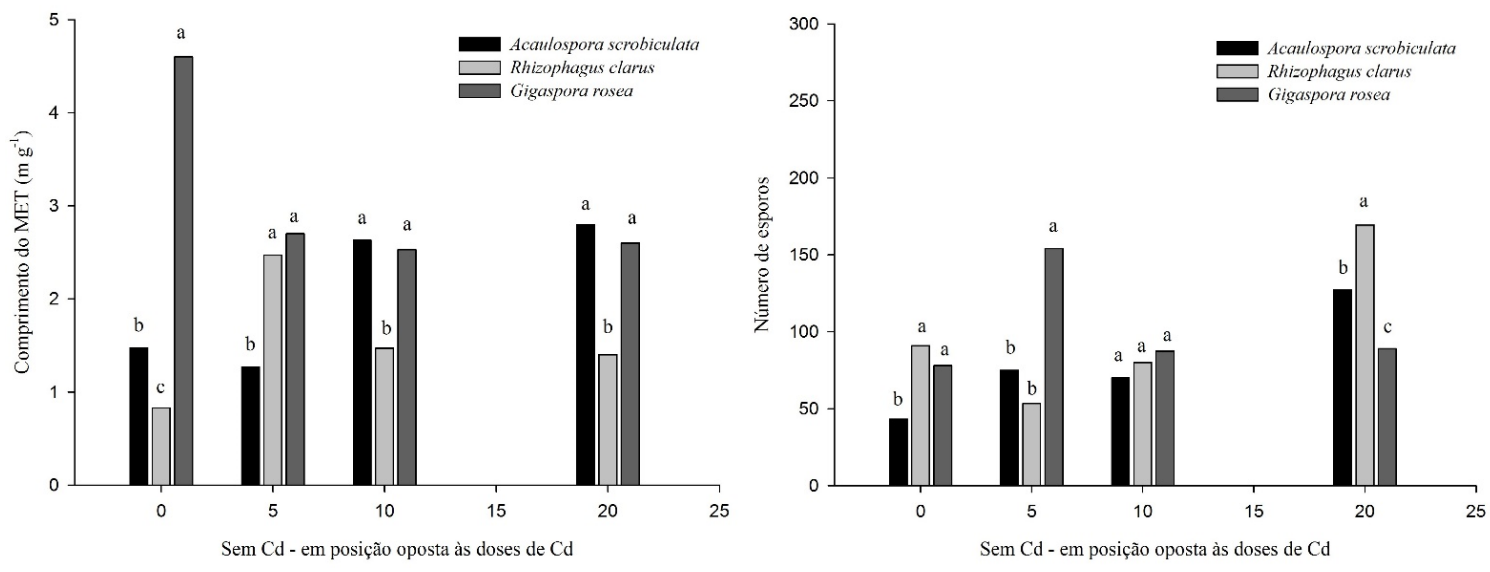

C

$\mathrm{D}$

Figura 4. Colonização radicular, dada pela porcentagem (A) e intensidade (B), comprimento de micélio extrarradicular (C) e número de esporos (D) das diferentes espécies de fungos micorrízicos arbusculares, no compartimento com solo não contaminado por $\mathrm{Cd}$. Médias seguidas por letras iguais na mesma dose não diferem estatisticamente entre si, por Tukey a $5 \%$ de significância.

Em experimentos conduzidos em áreas de mineração de zinco em Três Marias-MG, KlaubergFilho, Siqueira e Moreira (2002) demonstraram que a densidade e a riqueza das espécies de FMAs diminuíram de acordo com o aumento das concentrações de metais no solo, suprimindo as mais sensíveis e favorecendo as mais tolerantes. Para aquele trabalho, houve dominância das espécies $R$. clarus e $A$. mellea perante às outras dezenove espécies dos gêneros Acaulospora, Scutellospora, Glomus, Gigaspora e Entrophospora.
O comportamento diferenciado das espécies de FMAs deslocadas para a região não contaminada, oposta às doses de $\mathrm{Cd}\left(0,5,10,20 \mathrm{mg} \mathrm{kg}{ }^{-1}\right)$, encontram-se na figura 5.

Os valores da porcentagem de colonização radicular do milho (Figura $5 \mathrm{~A}$ ) proporcionado pela Acaulospora scrobiculata apresentaram decréscimo até a dose $10 \mathrm{mg} \mathrm{kg}^{-1} \mathrm{e}$, a partir daí, aumentaram de acordo com o aumento das doses, contrariando o resultado observado na figura $3 \mathrm{~A}$, onde houve redução da porcentagem de colonização ao longo das 
doses, demonstrando sensibilidade dessa espécie em doses elevadas de Cd. As espécies Rhizophagus clarus e Gigaspora rosea desenvolveram comportamento semelhante entre si, com redução dos valores ao longo das doses de Cd. Até a dose $10 \mathrm{mg} \mathrm{kg}^{-1}$, as espécies Acaulospora scrobiculata e Rhizophagus clarus apresentaram aumento da intensidade de colonização (Figura 5B), com redução em seguida.

O comprimento de micélio extrarradicular (Figura 5C) desenvolvido pela espécie Acaulospora scrobiculata pode ser descrito por regressão linear, com aumento dos valores ao longo das doses de $\mathrm{Cd}$, demonstrando maior tolerância dessa espécie em doses mais elevadas. Comportamento diferente foi observado para as demais espécies, que demonstraram efeito quadrático significativo, com maior sensibilidade da Gigaspora rosea em relação à Rhirophagus clarus.

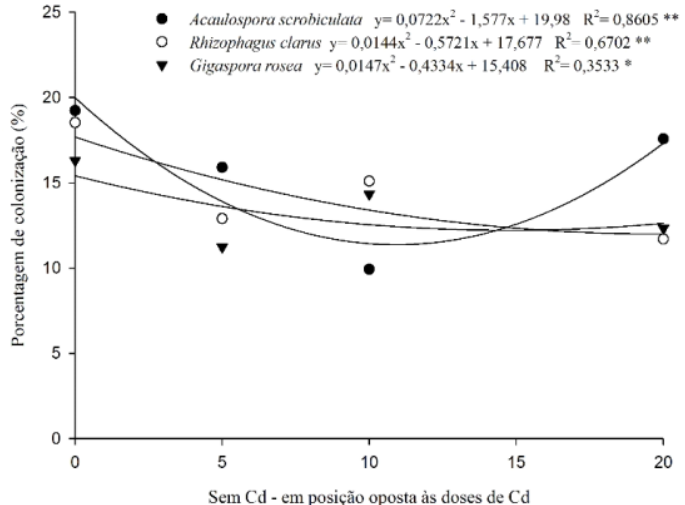

A

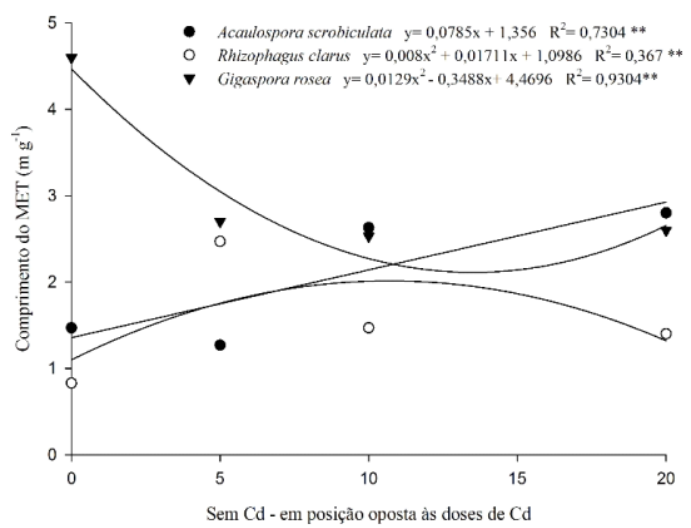

$\mathrm{C}$

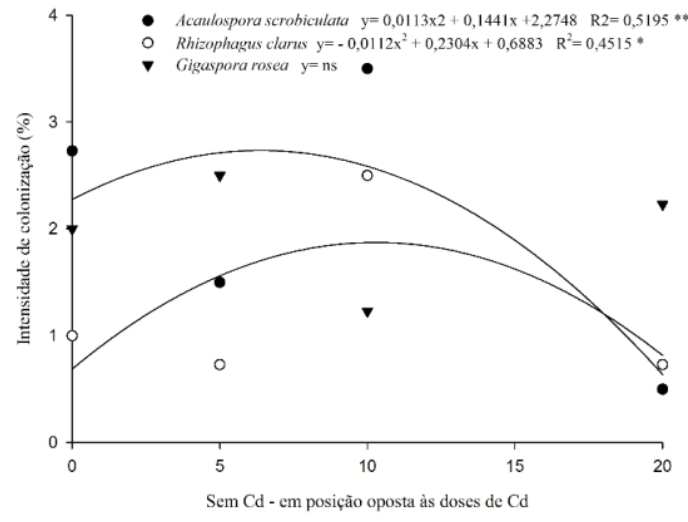

B

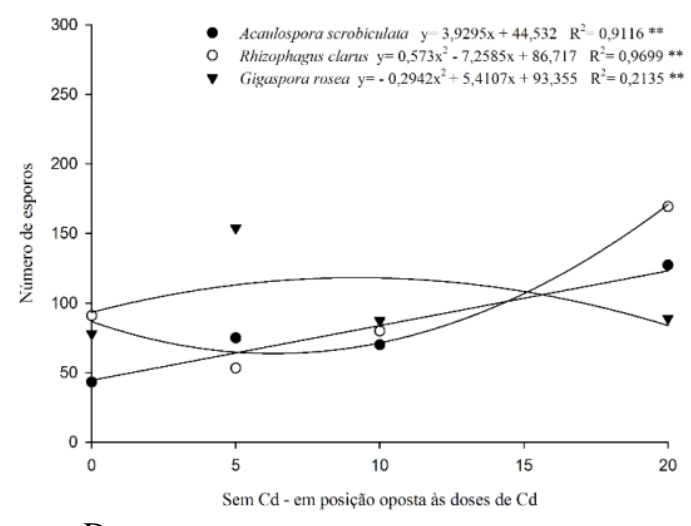

$\mathrm{D}$

Figura 5. Comportamento das espécies Acaulospora scrobiculata, Rhizophagus clarus e Gigaspora rosea deslocadas para a região não contaminada, oposta às doses de Cd. A - Porcentagem de colonização. B - Intensidade de colonização. $\mathrm{C}-$ Comprimento do micélio extrarradicular. $\mathrm{D}-$ Número de esporos. $*=\mathrm{p}<0,05 ; * *=\mathrm{p}<0,01$; ns $=$ não significativo. 
Para número de esporos (Figura 5D), Acaulospora scrobiculata apresentou comportamento descrito como regressão linear positiva e significativa, com elevação dos valores ao longo das doses de $\mathrm{Cd}$. A espécie Rhizophagus clarus também apresentou maior produção de esporos ao longo das doses, comportamento contrário ao observado para

Gigaspora rosea, a qual apresentou redução do número de esporos na última dose.

\section{CONCLUSÕES}

Todas as espécies de FMAs, Rhirophagus clarus, Gigaspora rosea e Acaulospora scrobiculata, apresentaram comportamento diferenciado na produção de propágulos (micélio extrarradicular e esporos) e formação de micorriza (intensidade e porcentagem de colonização), em relação ao aumento das doses de Cd.

Todas as espécies de FMAs produziram crescimento micelial em solos contaminados e não contaminados por $\mathrm{Cd}$, comprovando-se a capacidade de sobrevivência dessas espécies e de formação de micorriza em milho cultivado até $20 \mathrm{mg} \mathrm{kg}^{-1} \mathrm{de} \mathrm{Cd}$.

\section{AGRADECIMENTOS}

Ao CNPq e Fapemig, pelo apoio.

\section{REFERÊNCIAS}

ALVARENGA, M. A. R. Tomate: produção em campo, em casa-de-vegetação e em hidroponia, 2.a. ed. UFLA: Lavras, 2013. 455 p.

AMIR, H.; LAGRANGE, A.; HASSAÏNE, N.; CAVALOC, Y. Arbuscular mycorrhizal fungi from New Caledonian ultramafic soils improve tolerance to nickel of endemic plant species. Mycorrbiza, v. 23, p. 585-595, n. 7, 2013.

ANDRADE, S. A. L.; GRATÃO, P L.; AZEVEDO, R. A; SILVEIRA, A. P. D.;SCHIAVINATO, M. A.; MAZZAFERA, P. Biochemical and physiological changes in jack bean under mycorrhizal symbiosis growing in soil with increasing $\mathrm{Cu}$ concentrations. Environmental and Experimental Botany, v.68, p.198-207, 2009.
BETHLENFALVAY, G.J.; PACOVSKY, R.S.; BRONWN, M.S. Measurement of mycorrhizal infection in soybeans. Soil Science Society of America Journal, v.45, p. 871-875, 1981.

BURAK, D.L.; FONTES, M.P.F.; SANTOS, N.T.; MONTEIRO, L.V.S.; MARTINS, E.S.; BECQUER, T. Geochemistry and spatial distribution of heavy metals in Oxisols in a mineralized region of the Brazilian Central Plateau. Geoderma, v.160, p.131-142, 2010.

CABRAL, L; SIQUEIRA, J. O; SOARES. C. R. F. S.; PINTO, J. E. B. P. Retenção de metais pesados em micélio de fungos micorrízicosarbusculares. Química Nova, São Paulo, v. 33, n.1, p. 25-29, 2010.

CARDOSO, E.J.B.N.; CARDOSO, I. M.; NOGUEIRA, M. A.; BARRETA, C. R. D. M.; PAULA, A, M. Micorrizas arbusculares na aquisição de nutrientes pelas plantas. In: SIQUEIRA, J.O.; SOUZA, F. A; CARDOSO, E.J.B.N.; TSAI, S. M. Micorrizas: 30 anos de pesquisas no Brasil. Lavras: Ed. UFLA, 2010. p. 153-214.

COBBETT, C.; GOLDSBROUGH, P. Phytochelatins and Metallothioneins: Roles in Heavy Metal Detoxification and Homeostasis. Annual Reviews Plant Physiology, v.53, p.159-82, 2002.

COLODETE, C.M.; DOBBSS, L.B.; RAMOS, A.G. Aplicação de micorrizas arbusculares na recuperação de áreas impactadas. Natureza online, v.12, p.31-37, 2014.

CRUZ, A.F; MARTINS, M.A. Efeito de fungos micorrizicos arbusculares e doses de $\mathrm{N}$ sobre plantas cultivadas em sistema de consorcio. Revista Ceres. v. 45, p. 41-54, 1998.

CUNHA, K. P. V.; NASCIMENTO, C. W. A.; PIMENTEL, R. M. M.; ACCIOLY, A. M. A.; SILVA, A. J. Disponibilidade, acúmulo e toxidez de cádmio e zinco em milho cultivado em solo contaminado. Revista Brasileira de Ciências do Solo. Viçosa, v. 32, p. 1319-1328, 2008. 
DEL DUCCA, A. P.; MELLONI, E. G. P.; MELLONI, R.; MARTINS, F. B. Crescimento de Milhos Transgênico (Bt) e Não Transgênico Inoculados com Fungos Micorrízicos Arbusculares em Solo Contaminado por Cádmio. Revista Brasileira de Ciência do Solo, Viçosa, v. 39, n. 5, p. 1275-1281, 2015.

EMPRESA BRASILEIRA DE PESQUISA AGROPECUÁRIA - EMBRAPA. Centro Nacional de Pesquisa de Solos. Manual de métodos de análise de solo. 2.ed. Rio de Janeiro: Embrapa, 1997. 212p.

FERREIRA; D. F. Sisvar: a computer statistical analysis system. Ciência e Agrotecnologia, Lavras, v. 35, n.6, p. 1039-1042, 2011.

GARCIA, K. G. V.; GOMES, V. F. F.; ALMEIDA, A. M. M.; FILHO, P. F. M. Micorrizas arbusculares no crescimento de mudas de sabiá em um substrato proveniente da mineração de manganês. Revista Verde de Agroecologia e Desenvolvimento Sustentável, Pombal, v. 11, n. 2, p. 15-20, 2016.

GERDEMANN, J. W.; NICOLSON, T. H. Spores of mycorrhizal endogone species extracted from soil by wet sieving and decanting. Transactions of the British Mycological Society, Cambridge, v. 46, n. 2, p. 235-244, July 1963.

GIOVANETTI, M.; MOSSE, B. An evaluation of techniques for measuring vesicular arbuscular mycorrhizal infection in roots. New Phytologist, v. 84, p. 489-500. 1980

HALL, J. L. Cellular mechanisms for heavy metal detoxification and tolerance. Journal of Experimental Botany, Oxford,v. 53, p. 1-11, 2002.

JENTSCHKE, G.; WINTER, S.; GODBOLD, D.L. Ectomycorrhizas and cadmium toxicity. In: Norway spruce seedlings. Tree Physiology, v.19, p.23-30, 1999.

KERBAUY, G. B. Fisiologia vegetal. 2. ed. Rio de Janeiro: Guanabara Koogan, 2012.

KLAUBER-FILHO, O.; SIQUEIRA， J.O.; MOREIRA, F. S. M.; SOARES, C. R. F. S.; SILVA, S. Ecologia, função e potencial de aplicação de fungos micorrízicos arbusculares em condições de excesso de metais pesados. In: VIDAL-TORRADO, P.;
ALLEONI, L.R.F.; COOPER, M.; SILVA, A.P.; CARDOSO, E.J. Tópicos em Ciência do Solo. Viçosa: Sociedade Brasileira de Ciência do Solo, p.85-144, 2005.

KLAUBERG-FILHO， O.; SIQUEIRA， J. O.; MOREIRA, F. M. S. Fungos micorrízicos arbusculares em solos de área poluída com metais pesados. Revista Brasileira de Ciência do Solo, Viçosa, v. 26, n. 1, p. 125-134, 2002.

LAZAROTTO, M.; MUNIZ, M, F, B.; SANTOS, A, F. Detecção, transmissão, patogenicidade e controle químico de fungos em sementes de paineira (Ceiba speciosa). Summa Phytopathologica, Botucatu, v.36, n.2, p.134-139, 2010.

LINS, C.E.L.; CAVALCANTE, U. M. T.; SAMPAIO, E.V.S. B.; MESSIAS, A.S.; MAIA, L, C. Growth of mycorrhized seedlings of Leucaena leucocephala (Lam.) de Wit. in a copper contaminated soil. Applied Soil Ecology, v.31, p.181-185, 2006.

LU, A.; WANG, J.; QIN, X.; WANG, K.; HAN, P.; ZHANG, S. Multivariate and geostatistical analyses of the spatial distribution and origin of heavy metals in the agricultural soils in Shunyi, Beijing, China.Science ofthe Total Environment, v. 425, p. 66-74, 2012.

MELLONI, R.; CARDOSO, E. J. B.N. Quantificação de micélio extrarradicular de fungos micorrízicos arbusculares em plantas cítricas - I: método empregado. Revista Brasileira de Ciência do Solo, Viçosa, v. 23, n. 1, p. 53- 58, 1999.

MOREIRA, F. M. S.; SIQUEIRA, J. O. S. Microbiologia e Bioquimica do Solo. 2. ed. Lavras: UFLA, 2006. 730p.

MUGOUEI, R.; BORGHEI, M.; ARJMANDI, R. Phytoremediation of stable Cs from solutions by Calendula alata, Amaranthus chlorostachys and Chenopodium album.Ecotoxicology and Environmental Safety, v.74, p. 2036-2039, 2011.

NOQUEIRA, M. A. Micorrizas Arbusculares e Metais Pesados. In: SILVEIRA, A. P.D.; FREITAS, S.S. Microbiota do solo e qualidade ambiental. Campinas: Instituto Agronômico, 2007. p. 219-238. 729 p. 
NOGUEIRA, M. A; SOARES, C. R. F. S. Micorrizas arbusculares e elementos-traço. In: SIQUEIRA, J. O; SOUZA F. A; CARDOSO, E. J. B. N; TSAI, S. M. Micorrizas: 30 anos de pesquisa no Brasil. Lavras: UFLA, 2010. p. 475-501. 716p.

OLGUIN, E. J.; SANCHEZ-GALVAN, G.; PEREZ-PEREZ, T. Assessment of the phytoremediation potential of Salvinia minima Baker compared to Spirodela polyrrbizain high-strength organic wastewater. Water Air and Soil Pollution, v. 181, p. $135-147,2007$.

OSKARSSON, A.; LINDEN, A.; OLSSON, I. M.; PETERSSON, K. Cadmium in food chain and health effects in sensitive population groups. BioMetals, v.17, p.531-534, 2004.

PAIVA, H. N.; CARVALHO, J. G.; SIQUEIRA, J. O.; MIRANDA, J. R. P.; FERNANDES, A. R. Absorção de Nutrientes por mudas de ipê-roxo (Tabebuia impetiginosa em solução nutritiva contaminada por cádmio. Revista Árvore, Viçosa,v. 28, n. 2, p. 189-197, 2004.

PEREIRA, A. C. C.; RODRIGUES, A. C. D.; SANTOS, F. S.; GUEDES, J. N.; SOBRINHO, N. M. B. A. Concentração de metais pesados em espécies arbóreas utilizadas para revegetação de área contaminada. Revista Ciência Agronômica, v. 43, p. 641647, 2012.

PHILLIPS J. M.; HAYMAN D. S. Improved procedures for clearing roots and staining parasitic and vesicular-arbuscular mycorrhizal fungi for rapid assessment of infection. Transaction sof the British Mycological Society, v. 55, p. 158-161, 1970.
SILVA, S.; SIQUEIRA, J. O.; SOARES, C. R. F. S. Fungos micorrízicos no crescimento e na extração de metais pesados pela braquiária em solo contaminado. Pesquisa Agropecuária Brasileira, Brasília, v. 41, n. 12, p. 1749-1757, 2006.

SOARES, C. R. F. S.; CARNEIRO, M. A. C. Micorrizas arbusculares na recuperação de áreas degradadas. In: Siqueira J. O; SOUZA F. A; CARDOSO, E. J. B. N; TSAI, S. M. Micorrizas: 30 anos de pesquisa no Brasil. Lavras: UFLA, 2010. p. 441-474.

VALADARES, R. B. S.; MESCOLOTTI, D. L. C.; CARDOSO, E.J.B.N. Micorrizas. In: CARDOSO, E.J.B.N.; ANDREOTE, F. D. Microbiologia do Solo.Piracicaba: ESALQ, 2016. p. 179-196.

YANG, Y.; SONG, Y.; SCHELLER, H. V.; GHOSH. A.; BAN, Y, CHEN, H.; TANG, M. Community structure of arbuscular mycorrhizal fungi associated with Robinia pseudo acacia in uncontaminated and heavy metal contaminated soils. Soil Biology and Biochemistry, v. 86, p. 146-158, 2015.

ZHAO, F.J.; MCGRANT, S.P.; MERRINGTON, G. Estimates of ambient background concentrations of trace metals in soil for risk assessment. Environmental Pollution, v. 148, p. 221-229, 2007. 